\title{
Gerontology
}

\section{Relationship between Strength, Power and Balance Performance in Seniors}

\author{
Thomas Muehlbauer $^{a} \quad$ Carmen Besemer $^{b}$ Anja Wehrle ${ }^{c}$ Albert Gollhofer ${ }^{b}$ \\ Urs Granacher ${ }^{a}$ \\ ${ }^{a}$ Department of Training and Movement Science, University of Potsdam, Potsdam, ${ }^{\mathrm{b}}$ Institute of Sport and Sport \\ Science, Albert Ludwigs University Freiburg, and ' Department of Rehabilitative and Preventive Sports Medicine, \\ University Medical Center Freiburg, Freiburg, Germany
}

\section{Key Words}

Steady-state balance $\cdot$ Proactive/reactive balance .

Force production $\cdot$ Single/dual tasking $\cdot$ Cognitive/motor interference

\section{Abstract}

Background: Deficits in strength, power and balance represent important intrinsic risk factors for falls in seniors. Objective: The purpose of this study was to investigate the relationship between variables of lower extremity muscle strength/power and balance, assessed under various task conditions. Methods: Twenty-four healthy and physically active older adults (mean age: $70 \pm 5$ years) were tested for their isometric strength (i.e. maximal isometric force of the leg extensors) and muscle power (i.e. countermovement jump height and power) as well as for their steady-state (i.e. unperturbed standing, 10-meter walk), proactive (i.e. Timed Up \& Go test, Functional Reach Test) and reactive (i.e. perturbed standing) balance. Balance tests were conducted under single (i.e. standing or walking alone) and dual task conditions (i.e. standing or walking plus cognitive and motor interference task). Results: Significant positive correlations were found between measures of isometric strength and muscle power of the lower extremities ( $r$ values ranged between 0.608 and $0.720, p<0.01$ ). Hardly any significant associations were found between variables of strength, power and balance (i.e. no significant association in 20 out of 21 cases). Additionally, no significant correlations were found between measures of steady-state, proactive and reactive balance or balance tests performed under single and dual task conditions (all $p>0.05$ ). Conclusion: The predominately nonsignificant correlations between different types of balance imply that balance performance is task specific in healthy and physically active seniors. Further, strength, power and balance as well as balance under single and dual task conditions seem to be independent of each other and may have to be tested and trained complementarily.

Copyright @ 2012 S. Karger AG, Basel

\section{Introduction}

In older adults, high levels of strength, power and balance are important prerequisites for the independent and successful performance of activities of daily living. For example, standing up from a chair and climbing/descending stairs afford adequate levels of balance and

\section{KARGER}

Fax +41613061234

E-Mail karger@karger.ch

www.karger.com
(C) 2012 S. Karger AG, Basel

0304-324X/12/0586-0504\$38.00/0

Accessible online at:

www.karger.com/ger
Prof. Dr. Urs Granacher

Department of Training and Movement Science, University of Potsdam

Am Neuen Palais 10, Haus 12

DE-14469 Potsdam (Germany)

Tel. +49 331977 1543, E-Mail urs.granacher@uni-potsdam.de 
strength/power of the lower extremities to successfully complete these everyday tasks. In contrast, deficits in strength, power and balance represent important intrinsic risk factors for falls in seniors [1,2]. In fact, Pijnappels et al. [3] found an association between lower limb strength and the ability to prevent a fall after a gait perturbation in elderly individuals (mean age: $71 \pm 5$ years). Furthermore, Hausdorff et al. [4] reported that gait unsteadiness in terms of greater temporal and spatial stride-to-stride variability significantly differed between healthy older community-dwelling fallers (mean age: $82 \pm 5$ years) and nonfallers (mean age: $76 \pm 4$ years).

From a therapist's or practitioner's point of view, knowledge about the relationship between strength/ power of the lower extremity muscles and balance may be important for both the identification of elderly persons with a decreased performance level (i.e. an increased risk of sustaining fall-related injuries) and the development of fall-preventive training programs. More specifically, given the high number of fall-related injuries in older adults [5, 6], findings on potential associations between variables of lower extremity muscle strength/power and balance could provide scientific rationales for assessing the risk of falls and developing specifically tailored fall prevention and rehabilitation programs for seniors.

Potential associations between measures of isometric strength and muscle power of the lower extremities and different balance components have already been studied in healthy older adults. However, the reported correlations largely vary in size (low to high) and level of significance (nonsignificant to significant). For example, Pijnappels et al. [3] reported correlations between isometric strength (i.e. maximal isometric force (MIF), rate of force development (RFD) of the plantar flexors and knee extensors) and muscle power (i.e. countermovement jump (CMJ)) in seniors (mean age: $71 \pm 5$ years) ranging from $r=+0.29$ to +0.82 . Further, Spink et al. [7] assessed strength (i.e. maximal isometric ankle dorsiflexion, plantar flexion, inversion and eversion strength) and various types of balance (i.e. bipedal stance, alternate step test, 6-meter walking test) in older adults (mean age: $74 \pm 6$ years) and observed correlations ranging from $\mathrm{r}=-0.01$ to -0.41. Lastly, Shimada et al. [8] established correlations $(\mathrm{r}=-0.18$ to +0.34$)$ between steady-state (i.e. one-leg standing test, tandem walk test, 6-meter walking time) and proactive balance, i.e. Timed Up \& Go test (TUG) in elderly individuals (mean age: $80 \pm 7$ years). The reason for this discrepancy in the literature is probably to be found in the differing research designs applied (i.e. a large variety of methods for the assessment of strength, power and balance).

The above-cited studies $[3,7,8]$ together with additional studies [9-17] already extend the knowledge regarding the relationship between strength, power and balance performance. However, in these studies balance abilities were tested nonconclusively. In other words, steady-state balance (i.e. bipedal/one-leg standing test and walking tests) was compared with proactive (i.e. alternate-step test) or reactive (i.e. perturbed standing) balance but not the latter two types against each other. Furthermore, everyday balance situations have to be tested under single (i.e. standing or walking alone) and dual task conditions (i.e. walking while talking to somebody). The successful performance of dual task situations affords increased levels of attentional demand for the regulation of balance [18]. In fact, Granacher et al. [10] showed higher levels of postural sway and greater stride-to-stride variability during dual tasking (i.e. bipedal stance/10-meter walking test while counting backwards by 3) compared to single tasking (i.e. bipedal stance/10-meter walking test only) in seniors (mean age: $74 \pm 6$ years). Further, Lundin-Olsson et al. [19] observed that elderly subjects who stopped walking when talking had a significantly increased risk of sustaining a fall within the next 6 months. Knowledge about performance level in different types of balance (e.g. steadystate, proactive and reactive balance) and how they are related to each other, together with an understanding of how attentional demand (single vs. dual task situations) influences balance performance may be helpful for therapists to plan and design adequate intervention programs.

Therefore, the objectives of this study were threefold: first, to replicate earlier findings demonstrating associations between isometric strength, muscle power and various balance components (i.e. steady-state, proactive and reactive balance); second, to extend former findings by comparing reactive and proactive types of balance; third, to determine whether an association exists between various balance abilities performed under single and dual task conditions. Based on the currently available literature [3,7-17], significant relationships between measures of isometric strength and power of the lower extremities but not between steady-state, proactive and reactive balance, and between balance and strength/power measures are expected. Furthermore, we predict no significant correlations between several balance tests performed under single and dual task conditions [10]. 


\section{Methods}

\section{Participants}

Twenty-four healthy older adults participated in the study after experimental procedures were explained to them (sex: $13 \mathrm{fe}-$ male, 11 male; age: $70.3 \pm 4.6$ years; body height: $168.3 \pm 10.7$ $\mathrm{cm}$; body mass: $71.8 \pm 12.1 \mathrm{~kg}$; body mass index: $25.3 \pm 3.3$; everyday and sports-related physical activity: $5.4 \pm 3.7 \mathrm{~h} /$ week (e.g. walking, cycling, swimming, dancing, gardening, grocery shopping). Participants were recruited in the local community via advertisements in regional newspapers. The inclusion criteria were (1) adults 65 years or older, (2) independent community dwellers and (3) naïve in terms of strength, power and balance training. Participants were excluded if their medical history or physical examination revealed (1) a musculoskeletal, neurological and/or orthopedic disorder that might have affected their ability to perform strength, power and balance tests or (2) any other medical conditions that would limit participation (e.g. terminal illness or cognitive dysfunction). All participants provided written informed consent before the start of the study. The study was approved by the ethics committee of the Albert Ludwig University of Freiburg, Freiburg, Germany. All experiments were conducted according to the latest version of the Declaration of Helsinki.

\section{Testing Procedure}

Prior to testing, all subjects underwent a standardized 5-min warm-up consisting of bipedal and monopedal balance exercises as well as submaximal plyometrics. Measurements included (a) assessment of MIF of the leg extensors on a leg press, (b) analysis of $\mathrm{CMJ}$ height $\left(\mathrm{CMJ}_{\mathrm{H}}\right)$ and $\mathrm{CMJ}$ power $\left(\mathrm{CMJ}_{\mathrm{P}}\right)$ on a force platform, (c) testing of steady-state (i.e. unperturbed standing, 10-meter walk), reactive (i.e. perturbed standing) and proactive, i.e. TUG, Functional Reach Test (FRT) balance. Steady-state (i.e. unperturbed standing, 10-meter walk) and proactive (i.e. TUG) balance tests were performed under single (i.e. balance task alone) and dual task conditions, i.e. balance task plus cognitive (CI) and motor (MI) interference task.

\section{Strength Testing}

MIF was measured on a leg press, with the feet resting on a one-dimensional force platform (KistlerM type 9253B, Winterthur, Switzerland; measurement error: $\leq 0.5 \%$ ). Participants were positioned horizontally on the sledge of the leg press with hip and knee angles adjusted to $90^{\circ}$ and the ankle angle to $100^{\circ}$. The waist was fixed and participants were allowed to stabilize their upper body by holding on to handles attached to the leg press. Participants were instructed to avoid forced respiration during maximal efforts. Before the testing started, participants were asked to perform 3-5 submaximal isometric contractions to get accustomed to the testing procedure. Thereafter, each participant performed 3-4 leg press exercises with maximal voluntary effort lasting 3$5 \mathrm{~s}$ each. For each trial, participants were thoroughly instructed to extend their legs as forcefully and as fast as possible. The force signal perpendicular to the force plate was sampled at $500 \mathrm{~Hz}$. Force signals were converted analog to digital and stored on a computer. During later offline analysis, the best trial in terms of MIF was selected and the force signal was filtered with a digital fourth-order recursive Butterworth low-pass filter, using a cut-off frequency of $50 \mathrm{~Hz}$. MIF was calculated from the individual maximal isometric force development record. MIF was defined as the maximal voluntary force value of the force-time curve, determined under isometric condition. In terms of test-retest reliability, intraclass correlation coefficients (ICC) were calculated and amounted to ICC $=0.99$ for MIF.

\section{Power Testing}

Participants performed maximal vertical CMJs while standing on a three-dimensional force platform (Kistler type 9281A; measurement error: $\leq 0.2 \%)$. The vertical ground reaction force was sampled at $1,000 \mathrm{~Hz}$. During the CMJ, subjects stood in an upright position on the force plate and were instructed to begin the jump with a downward movement, which was immediately followed by a concentric upward movement, resulting in a maximal vertical jump. Subjects performed $3 \mathrm{CMJs}$ with a resting period of 1 min between jumps. For each of these trials, subjects were asked to jump as high as possible. The best trial in terms of maximal jump height was taken for further data analysis. Testretest reliability amounted to $\mathrm{ICC}=0.99$ and $\mathrm{ICC}=0.97$ for $\mathrm{CMJ}_{\mathrm{H}}$ and $\mathrm{CMJ}_{\mathrm{P}}$, respectively.

\section{Balance Testing}

Test conditions (e.g. room illumination, temperature and noise) were in accordance with recommendations for posturographic testing [20]. Steady-state balance was assessed by means of a balance platform (GKS 1000S, IMM, Mittweida, Germany). The balance platform consists of four uniaxial sensors measuring displacements of the center of pressure $(\mathrm{CoP})$ in the mediolateral and anteroposterior directions. The balance platform was firmly fixed on the floor. For experimental testing, participants were asked to stand (i.e. bipedal step stance) in erect position with hands placed on hips and gaze fixed on a cross on the nearby wall. Subjects were instructed to remain as stable as possible and to refrain from any voluntary movements during the trials. Prior to testing, participants performed two practice trials on the balance platform. Thereafter, three test trials were conducted. The best trial (least CoP displacements) was used for further analysis. Data were acquired for $30 \mathrm{~s}$ at a sampling rate of $40 \mathrm{~Hz}$ [20]. Total displacements of the $\mathrm{CoP}\left(\mathrm{CoP}_{\text {tot_s }}\right.$ in millimeters) as well as displacements of the $\mathrm{CoP}$ in the anteroposterior $\left(\mathrm{CoP}_{\mathrm{ap} \_s}\right.$ in millimeters) and mediolateral $\left(\mathrm{CoP}_{\mathrm{ml} \_s}\right.$ in millimeters) directions were computed and used as outcome measures. Test-retest reliability amounted to ICC $=0.87$, ICC $=0.71$ and ICC $=0.79$ for $\mathrm{CoP}_{\text {tot_s }}$, $\mathrm{CoP}_{\text {ap_s }}$ and $\mathrm{CoP}_{\mathrm{ml} \_}$, respectively.

During the reactive balance test, participants stood in bipedal step stance on a two-dimensional balance platform (Posturomed, Haider, Bioswing, Pullenreuth, Germany). The platform is mounted on four springs and is free to move in the transversal, mediolateral and anteroposterior directions. The maximal natural frequency of the Posturomed is below $3 \mathrm{~Hz}$. The mechanical constraints and the reliability of the system were described earlier [21]. If the platform is in neutral position, the maximum range of motion in the mediolateral and anteroposterior directions amounts to $70 \mathrm{~mm}$, respectively. Mediolateral perturbation impulses were applied in order to investigate the reactive postural control of the participants. Therefore, the platform was moved 2.5 $\mathrm{cm}$ from the neutral position in the mediolateral direction, where it was magnetically fixed. For experimental testing, participants were asked to stand (i.e. bipedal step stance) in the erect position with their hands placed on their hips and gaze fixed on a cross on the nearby wall. Three to five trials helped participants to get ac- 
customed to the measuring device. After investigators visually controlled the position of the subjects, the mediolateral perturbation impulse was unexpectedly applied by detaching the magnet. The platform suddenly accelerated in the medial direction. The participants' task was to damp the oscillating platform by balancing on the Posturomed. Summed oscillations of the platform in the mediolateral $\left(\mathrm{SO}_{\mathrm{ml} \_\mathrm{r}}\right)$ and anteroposterior $\left(\mathrm{SO}_{\mathrm{ap} \_\mathrm{r}}\right)$ directions were assessed by means of a joystick-like 2D potentiometer (Megatron) which was connected to the platform. The potentiometer measured the position of the platform in degrees. The signal was differentiated, rectified and integrated over the 10 -second test interval. Three trials were performed. The best trial (least oscillations in the mediolateral direction) was used for further analysis. The test-retest reliability amounted to ICC $=0.69$ and ICC $=0.40$ for $\mathrm{SO}_{\text {ap_r }}$ and $\mathrm{SO}_{\text {ap_r }}$, respectively.

Gait speed was determined during a 10 -meter walking test. Participants walked with their own footwear at self-selected speeds, initiating and terminating each walk a minimum of $1 \mathrm{~m}$ before and after the 10-meter walkway to allow sufficient distance to accelerate to and decelerate from a steady state of ambulation across the walkway. One practice and one test trial were performed. Time was recorded with a stopwatch to the nearest $0.01 \mathrm{~s}$. Before testing, a trained evaluator gave standardized verbal instructions regarding the test procedure. Participants were instructed to walk $12 \mathrm{~m}$ at their preferred speed. On the command 'ready-set-go', the participants started their walk. The stopwatch was started after the 1-meter acceleration phase and stopped after the 10-meter walking distance. Participants kept on walking for another meter. The ICC values for gait velocity ranged between 0.59 and 0.79 depending on task condition (i.e. single and dual tasking). The validity of the parameter gait velocity was established by Montero-Odasso et al. [22] when testing communitydwelling older adults.

The TUG was used as described by Podsiadlo and Richardson [23]. Participants were asked to perform the TUG at their self-selected normal speed. One practice and one test trial were performed. Time was recorded with a stopwatch to the nearest $0.01 \mathrm{~s}$. Before testing, a trained evaluator gave standardized verbal instructions regarding the test procedures. Participants were seated and instructed to walk $3 \mathrm{~m}$, turn around, walk back to the chair and sit down. The stopwatch was started on the command 'readyset-go' and stopped as the participant sat down. The TUG showed excellent test-retest reliability $(\mathrm{ICC}=0.99)$ in older adults [23].

Proactive balance was further assessed by means of the FRT [24]. For this purpose, we constructed a moveable sliding apparatus which allowed determination of the maximal distance one can reach forward beyond arm's length while maintaining a fixed base of support in the standing position [25]. One practice trial was performed to familiarize participants with the FRT. Trials 2-4 were averaged and taken for further analysis. The FRT showed excellent test-retest reliability (ICC $=0.92$ ) in older adults [24]. The validity of the FRT was established by Newton [26] when testing healthy community-dwelling older adults.

\section{CI and CM Task}

Performance during steady-state (i.e. unperturbed standing, 10-meter walking) and proactive (i.e. TUG) balance tasks were also examined while performing a concurrent attention-demanding CI and MI task. The CI task was an arithmetic task, in which the participants recited out loud serial subtractions by 3 starting
Table 1. Performance variables for strength, power, and balance measurements

\begin{tabular}{|c|c|}
\hline $\begin{array}{l}\text { Isometric strength } \\
\text { MIF, N }\end{array}$ & $824.5 \pm 343.4$ \\
\hline \multicolumn{2}{|l|}{ Power } \\
\hline $\mathrm{CMJ}_{\mathrm{H}}, \mathrm{cm}$ & $17.0 \pm 4.4$ \\
\hline $\mathrm{CMJ}_{\mathrm{P}}, \mathrm{W} / \mathrm{kg}$ & $27.1 \pm 4.8$ \\
\hline \multicolumn{2}{|l|}{ Dual task balance } \\
\hline $\mathrm{CoP}_{\text {tot_ci }}, \mathrm{mm}$ & $1,025.3 \pm 136.4$ \\
\hline CoP tot_mi, $\mathrm{mm}$ & $947.7 \pm 89.5$ \\
\hline Gait velocity_ci, m/s & $1.2 \pm 0.2$ \\
\hline Gait velocity_mi, $\mathrm{m} / \mathrm{s}$ & $0.9 \pm 0.2$ \\
\hline $\mathrm{TUG}_{\mathrm{ci}}, \mathrm{s}$ & $9.8 \pm 1.8$ \\
\hline $\mathrm{TUG}_{\mathrm{mi}}, \mathrm{s}$ & $15.4 \pm 4.1$ \\
\hline \multicolumn{2}{|c|}{ Single task steady-state balance } \\
\hline $\mathrm{CoP}_{\text {tot_s }}, \mathrm{mm}$ & $885.0 \pm 277.3$ \\
\hline $\mathrm{CoP}_{\text {ap_s }}, \mathrm{mm}$ & $629.9 \pm 233.8$ \\
\hline $\mathrm{CoP}_{\mathrm{ml} \_s}, \mathrm{~mm}$ & $495.5 \pm 141.5$ \\
\hline Gait velocity, $\mathrm{m} / \mathrm{s}$ & $1.3 \pm 0.2$ \\
\hline \multicolumn{2}{|c|}{ Single task reactive/proactive balance } \\
\hline $\mathrm{SO}_{\text {ap_r_r }}, \mathrm{mm}$ & $1,015.1 \pm 83.5$ \\
\hline $\mathrm{SO}_{\mathrm{ml} \_\mathrm{r}}, \mathrm{mm}$ & $2,234.5 \pm 107.7$ \\
\hline TUG, s & $8.2 \pm 1.3$ \\
\hline $\mathrm{FRT}, \mathrm{cm}$ & $32.2 \pm 7.0$ \\
\hline
\end{tabular}

Values are means \pm SDs.

from 100 [27]. The MI task required participants to hold two interlocked sticks steady in front of the body. One stick was held in each hand with the elbow in $90^{\circ}$ flexion. Each stick had a ring at the end with a diameter of $4 \mathrm{~cm}$ and the rings were interlocked [28]. Participants were advised not to let the rings touch each other. When the dual task methodology was used, participants were instructed to give equal priority to both tasks in order to create real-life conditions [29]. All task conditions were performed in a counterbalanced order.

\section{Statistical Analyses}

Data are presented as group mean values \pm standard deviations (SD). Associations of strength, power and balance variables were assessed using the Pearson product-moment correlation coefficient. Associations are reported by their correlation coefficient ( $r$ value), level of significance ( $p$ value) and the amount of variance explained ( $r^{2}$ value). Values of $r=0.10$ indicate a small, $r=0.30$ a medium and $r=0.50$ a large-size correlation [i.e. effect size (ES)] [30]. The significance level was set at $\alpha=5 \%$. All analyses were performed using Statistical Package for Social Sciences (SPSS) version 19.0.

\section{Results}

For all variables, means \pm SDs are presented in table 1 . 
Table 2. Correlation between variables of steady-state, proactive and reactive balance in seniors

\begin{tabular}{|c|c|c|c|}
\hline \multirow{3}{*}{$\begin{array}{l}\text { Single task reactive/ } \\
\text { proactive balance }\end{array}$} & \multicolumn{3}{|c|}{ Single task steady-state balance } \\
\hline & \multicolumn{2}{|l|}{ standing } & \multirow{2}{*}{$\frac{\text { walking }}{\text { gait velocity, m/s }}$} \\
\hline & $\mathrm{CoP}_{\text {ap_s }}, \mathrm{mm}$ & $\mathrm{CoP}_{\mathrm{ml} \_\mathrm{s}}, \mathrm{mm}$ & \\
\hline \multicolumn{4}{|l|}{ Reactive balance } \\
\hline $\mathrm{SO}_{\mathrm{ap} \_r}, \mathrm{~mm}$ & -0.025 & 0.051 & 0.046 \\
\hline $\mathrm{SO}_{\mathrm{ml} \_\mathrm{r}}, \mathrm{mm}$ & 0.010 & -0.082 & 0.014 \\
\hline \multicolumn{4}{|l|}{ Proactive balance } \\
\hline FRT, cm & 0.160 & 0.181 & -0.098 \\
\hline TUG, s & 0.144 & 0.156 & -0.303 \\
\hline
\end{tabular}

Table 3. Correlation between variables of strength, power and balance in seniors

\begin{tabular}{lccc}
\hline $\begin{array}{l}\text { Single task balance } \\
\text { performance }\end{array}$ & \multicolumn{3}{c}{ Strength and power performance } \\
\cline { 2 - 4 } & $\mathrm{MIF}, \mathrm{N}$ & $\mathrm{CMJ}_{\mathrm{H}}, \mathrm{cm}$ & $\mathrm{CMJ}_{\mathrm{P}}, \mathrm{W} / \mathrm{kg}$ \\
\hline $\mathrm{CoP}_{\mathrm{ap} \_s}, \mathrm{~mm}$ & 0.266 & 0.111 & 0.199 \\
$\mathrm{CoP}_{\mathrm{ml}}, \mathrm{mm}$ & 0.357 & 0.214 & 0.375 \\
$\mathrm{SO}_{\mathrm{ap} \_\mathrm{r}}, \mathrm{mm}$ & -0.152 & -0.173 & 0.026 \\
$\mathrm{SO}_{\mathrm{ml}, \mathrm{r}} \mathrm{mm}$ & -0.151 & -0.123 & 0.013 \\
FRT, cm & $0.468^{*}$ & 0.268 & 0.137 \\
TUG, s & -0.101 & -0.330 & -0.353 \\
Gait velocity, m/s & 0.028 & -0.117 & -0.214 \\
\hline
\end{tabular}

${ }^{*} \mathrm{p}<0.05$.

Table 4. Correlation between balance tests performed under single (ST) and dual task (DT) conditions in seniors

\begin{tabular}{llcc}
\hline Walking & \multicolumn{3}{l}{ Standing $\left(\mathrm{CoP}_{\text {tot }}, \mathrm{mm}\right)$} \\
\cline { 2 - 4 } & $\mathrm{ST}$ & $\mathrm{DT}-\mathrm{CI}$ & $\mathrm{DT}-\mathrm{MI}$ \\
\hline TUG, s & 0.152 & 0.324 & -0.081 \\
Gait velocity, $\mathrm{m} / \mathrm{s}$ & 0.276 & 0.017 & 0.154 \\
\hline
\end{tabular}

\section{Strength and Power Performance}

Significant positive correlations were found between MIF and $\mathrm{CMJ}_{\mathrm{H}}$ (fig. 1) as well as between MIF and $\mathrm{CMJ}_{\mathrm{P}}$ (data not shown). The respective $\mathrm{r}$ values amounted to $+0.720(\mathrm{p}<0.01)$ and $+0.608(\mathrm{p}<0.01)$, which is indicative of a large ES. This resulted in an explained variance of 52 and $37 \%$, respectively.

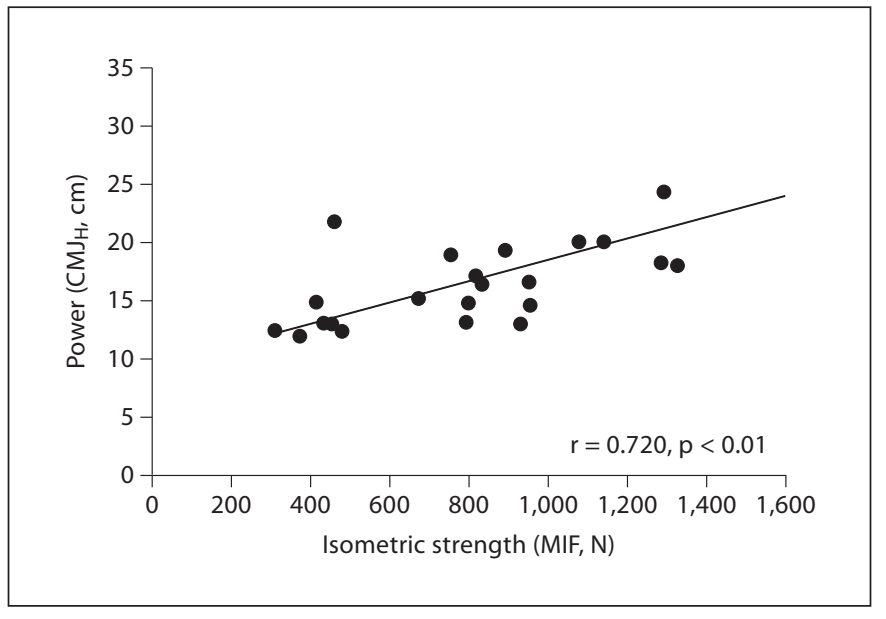

Fig. 1. Scatterplot of the relationship between isometric strength (MIF in newtons) and power $\left(\mathrm{CMJ}_{\mathrm{H}}\right.$ in centimeters) of lower extremity muscles.

\section{Balance Performance}

No statistically significant correlations were found between variables of steady-state and reactive balance in 12 out of 12 cases. The respective $r$ values ranged from +0.010 to -0.082 , which corresponds to a small ES (table 2). The explained proportion of variance was $\leq 1 \%$. In addition, no statistically significant correlations were found between steady-state and proactive balance. The respective $r$ values ranged from -0.098 to -0.303 (i.e. small to medium ESs) with an explained proportion of variance ranging from 1 to $9 \%$ (table 2).

\section{Strength, Power and Balance Performance}

No significant correlations were observed between variables of lower extremity muscle strength, power and balance in 20 out of 21 cases (except for the comparison of MIF with FRT: $r=0.468, p<0.05)$. The respective $r$ values ranged between 0.013 and +0.468 , which is indicative of small to medium ESs (table 3). Based on $r^{2}$, only a small proportion of the variance was explained (0-22\%).

\section{Balance Performance under Single and Dual Task \\ Conditions}

No significant correlations were observed between variables of balance performed under single and dual task conditions. The respective $r$ values ranged between 0.017 and +0.324 , which corresponds to small to medium ESs (table 4 ). Based on $\mathrm{r}^{2}$, only a small proportion of the variance was explained (1-10\%). 
Fig. 2. Relationship between strength, power and balance in older adults as reported in the literature.

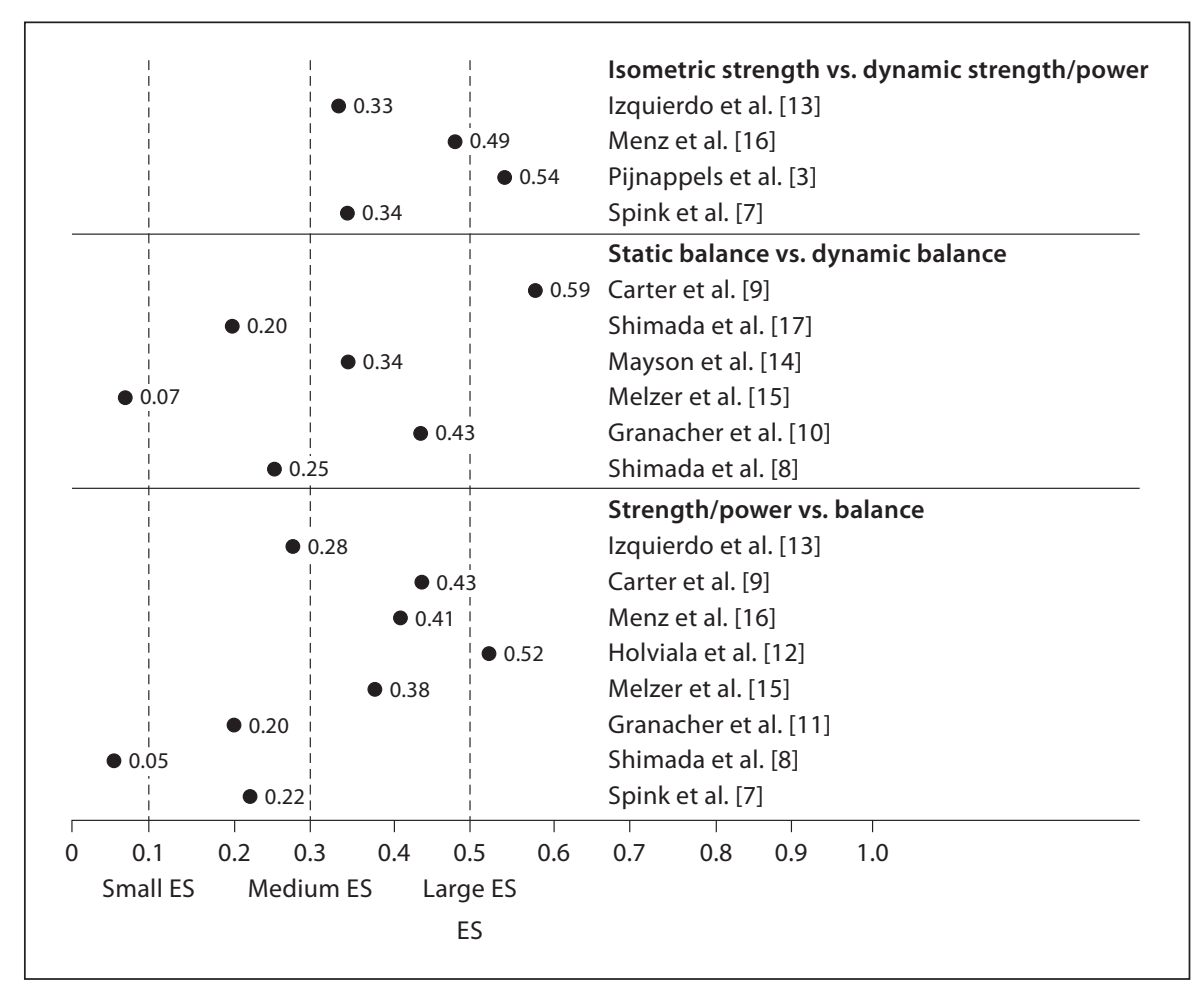

Additionally, no gender-specific differences were observed in the direction and size of correlations between the variables of strength, power and balance as well as between balance tests performed under single and dual task conditions. Yet, the level of significance (i.e. the correlation between strength and power measures) was no longer given, probably due to the reduction in sample size (i.e. $\mathrm{n}=11$ men and $\mathrm{n}=13$ women vs. a total sample size of $\mathrm{n}=24)$.

\section{Discussion}

Using correlative analyses, this study investigated potential associations between different measures of strength, power and balance in healthy and physically active older adults. The main findings of this study can be summarized as follows: (1) statistically significant correlations were observed between variables of isometric strength and power of lower-extremity muscles; (2) no statistically significant correlations were detected between parameters of steady-state, proactive and reactive balance; (3) hardly any statistically significant associations (except for 1) were found between measures of strength, power and balance, and (4) no statistically sig- nificant correlations were detected between balance tests performed under single and dual task conditions.

\section{Strength and Power Performance}

The present results are in accordance with the literature regarding the association between isometric strength and power variables of lower-extremity muscles. For example, Izquierdo et al. [13] compared isometric strength and power measures in healthy and habitually physically active men (mean age: $71 \pm 5$ years). For this purpose, the participants performed maximal isometric leg extensions on a leg press and CMJs. $\mathrm{CMJ}_{\mathrm{H}}$ showed statistically significant correlations (i.e. medium ES) with RFD of the leg extensors during maximal isometric contraction (fig. 2). Furthermore, Pijnappels et al. [3] reported statistically significant correlations (i.e. medium to large ES) between MIF/RFD of the plantar flexor and knee extensors and $\mathrm{CMJ}_{\mathrm{H}}$ in healthy older adults (mean age: $71 \pm 5$ years) ranging from $r=+0.29$ to +0.82 (fig. 2). Lastly, Menz et al. [16] and Spink et al. [7] found significant correlations (i.e. medium ES) between various measures of strength performance (i.e. maximal isometric ankle dorsiflexion, plantar flexion, inversion or eversion) in healthy community-dwelling adults aged 65 years and older (fig. 2). 
From a practitioner's perspective, these findings may indicate that training-induced strength adaptations are not necessarily task specific in physically active older adults. In other words, gains in isometric muscle strength could be transferred at least to a certain extent to ballistic conditions and vice versa. In fact, Henwood et al. [31] were able to show that resistance training conducted on weight machines significantly increased both muscle strength (i.e. MIF of the leg extensors) and muscle power (i.e. muscle power of the leg extensors) in healthy older adults (age range: 65-84 years). This finding could be beneficial for therapists and practitioners in terms of the development and application of effective resistance and power training programs for the prevention and rehabilitation of falls.

\section{Balance Performance}

Our findings are in accordance with earlier studies that scrutinized this issue in healthy older adults (fig. 2). In fact, Melzer et al. [15] applied the bipedal upright stand test (i.e. steady-state balance) and the limits of stability test (i.e. proactive balance) in healthy older adults with a mean age of $78 \pm 6$ years. Those authors could not find any statistically significant correlations (i.e. small ES) and concluded that different postural control mechanisms may be used to control balance, during quiet standing and during reaching movements [15]. This finding is reinforced by a recent study by Shimada et al. [8] who investigated this issue in healthy seniors aged $80 \pm 7$ years. Those authors detected associations between measures of steady-state (i.e. one-leg stance, tandem walk, 6-meter walking time) and proactive balance (i.e. TUG) that corresponded to small to medium ESs. Therefore, it seems plausible to argue that steady-state and proactive balance are regulated by different neuromuscular mechanisms. Based on the results of the present study, this assumption can be supported and extended in terms of the comparison between steady-state and reactive balance. Hence, we did not detect significant associations between unperturbed and perturbed standing or between 10-meter walking and perturbed standing.

These results may have functional implications for future directions in assessment of risk of falls as well as in planning and developing adequate training programs to counteract intrinsic risk factors for falls (e.g. balance deficits) in healthy older adults. Based on our findings, it can be hypothesized that different neuromuscular mechanisms are responsible for the regulation of steady-state, proactive and reactive balance. Given that fall-related injuries primarily occur during ambulation and thus dur- ing proactive, reactive and steady-state (i.e. walking) balance conditions in seniors [32], the risk of falls should be assessed specifically under these conditions to identify potential balance problems. From a fall-preventive point of view, our results indicate that steady-state, proactive, and reactive postural control appear to be independent of each other in healthy and physically active seniors and may have to be trained complementarily during balance training. However, this hypothesis needs to be verified by longitudinal (i.e. interventional) studies.

\section{Strength, Power and Balance Performance}

In the present study, hardly any significant associations (except one) were found between variables of lower extremity muscle strength, power and balance in healthy and physically active seniors. This is in accordance with recent studies comparing various measures of strength, power and balance (fig. 2). For example, Granacher et al. [11] studied the relationship between MIF and RFD of the leg extensors and reactive balance (i.e. the ability to compensate for gait perturbations during walking on a treadmill) in physically active elderly men (mean age: $67 \pm 4$ years). Those authors could not evidence any significant associations between these variables (i.e. small ES). Similar results were reported by Spink et al. [7] for strength (i.e. maximal isometric ankle dorsiflexion, plantar flexion, inversion, eversion, knee extension) and steady-state/ proactive balance (i.e. bipedal stance, $6 \mathrm{~m}$ walking test, alternate step test) in older adults (age range: 65-93 years) and by Shimada et al. [8] for strength (i.e. chair rise time) and steady-state balance (i.e. one-leg stance or tandem walk) in healthy seniors aged $80 \pm 7$ years. Based on our results and the findings reported in the literature $[7,8,11]$, it seems plausible to argue that lower-extremity muscle strength, power and balance are independent of each other in healthy and physically active seniors and may have to be trained complementarily for fall prevention purposes.

In contrast to the above-described findings, earlier studies on the relationship between strength, power and balance found significant correlations (fig. 2). In fact, Carter et al. [9] established a significant association (i.e. medium ES) between MIF of the knee extensors and performance on the figure-of-eight walking test (i.e. proactive balance) in older community-dwelling women (mean age: $69 \pm 3$ years). Similar results were obtained by Menz et al. [16] for MIF of the knee extensors and 6-meter walking speed in healthy community-dwelling older adults (age range: 62-96 years) and by Holviala et al. [12] for MIF of the leg extensors and 10-meter walking time in healthy 
community-dwelling older adults aged $\geq 65$ years. The reason for this discrepancy in the literature is probably to be found in the differing testing methodology applied (i.e. large variety of methods for the assessment of strength, power and balance). Therefore, further studies are necessary to elucidate whether strength, power and balance performances of healthy older adults are independent or dependent of each other.

\section{Balance Performance under Single and Dual Task Conditions}

In this study, no significant associations were detected between balance performance under single and dual task conditions in healthy and physically active older adults. This is in line with a recent study by Granacher et al. [10], who also investigated this issue in healthy and physically active community-dwelling seniors (mean age: $74 \pm 6$ years). As in the present study, no significant associations were observed between different variables of quiet standing and 10-meter walking performed under single and dual task conditions. Further, these authors hypothesized that single and dual task balance performances are independent of each other [10].

This result may have functional implications for future directions in assessing the of risk of falls as well as in planning and developing adequate training programs to counteract intrinsic risk factors for falls in healthy older adults. Given that dual task situations are common in daily life (e.g. walking while talking), the risk of falls should be assessed specifically under dual task conditions to identify potential balance problems. As to the prevention of falls, our results indicate that standing and walking erect under single and dual task conditions seem to be independent of each other in healthy older adults and may have to be trained complementarily during balance training. However, this hypothesis needs to be verified by longitudinal (i.e. interventional) studies.

We acknowledge that this study has some limitations that warrant discussion. More specifically, the participants included in this study were healthy physically active older adults. Therefore, caution is needed when generalizing the present findings to other populations (e.g. mobility-limited subjects or patients). In addition, the results are specific to the testing methodology used to assess isometric strength (i.e. MIF of the leg extensors) and power (i.e. CMJ) as well as steady-state (i.e. unperturbed standing, 10-meter walk), proactive (i.e. TUG, FRT) and reactive (i.e. perturbed standing) balance in this study. These measures may not represent all components of strength, power and balance. Therefore, caution is needed when generalizing the present findings to other testing situations.

\section{Conclusions}

Based on the results of this study, steady-state, proactive and reactive balance appear to be independent of each other in healthy and physically active older adults. Given that fall-related injuries primarily occur during ambulation and thus during proactive, reactive and steady-state (i.e. walking) balance in older adults [32], the risk of falls should be preferably carried out under these conditions to identify potential balance deficits. From the perspective of prevention, our results imply that steadystate, proactive and reactive exercises should be incorporated into balance training programs aiming at preventing falls. Furthermore, the strong associations between the isometric strength and power of the lower extremity muscles imply that gains made in one variable (e.g. MIF of the leg extensors) after training may be associated with a change in performance in other variables (e.g. $\mathrm{CMJ}_{\mathrm{H}}$ ). Thus, increases in isometric lower extremity muscle strength following resistance training can be transferred at least to a certain extent to an improved jump performance and vice versa. The nonsignificant findings regarding the associations between strength, power and balance as well as between balance performance under single and dual task conditions indicate that these important capacities may be unrelated in healthy older adults and may have to be trained complementarily for preventing falls.

\footnotetext{
References $>_{1}$ Maki BE, Holliday PJ, Topper AK: A prospective study of postural balance and risk of falling in an ambulatory and independent elderly population. J Gerontol 1994;49:M72M84.

2 Rubenstein LZ, Josephson KR: The epidemiology of falls and syncope. Clin Geriatr Med 2002;18:141-158.

3 Pijnappels M, van der Burg PJ, Reeves ND, van Dieen JH: Identification of elderly fallers by muscle strength measures. Eur J Appl Physiol 2008;102:585-592.

-4 Hausdorff JM, Edelberg HK, Mitchell SL, Goldberger AL, Wei JY: Increased gait unsteadiness in community-dwelling elderly fallers. Arch Phys Med Rehabil 1997;78:278283.
} 
5 Luukinen H, Koski K, Hiltunen L, Kivela SL: Incidence rate of falls in an aged population in northern Finland. J Clin Epidemiol 1994; 47:843-850.

6 Rubenstein LZ: Falls in older people: epidemiology, risk factors and strategies for prevention. Age Ageing 2006;35(suppl 2):ii37ii41.

7 Spink MJ, Fotoohabadi MR, Wee E, Hill KD, Lord SR, Menz HB: Foot and ankle strength, range of motion, posture, and deformity are associated with balance and functional ability in older adults. Arch Phys Med Rehabil 2011;92:68-75.

-8 Shimada H, Tiedemann A, Lord SR, Suzukawa M, Makizako H, Kobayashi K, Suzuki T: Physical factors underlying the association between lower walking performance and falls in older people: a structural equation model. Arch Gerontol Geriatr 2011;53: 131-134.

-9 Carter ND, Khan KM, Mallinson A, Janssen PA, Heinonen A, Petit MA, McKay HA: Knee extension strength is a significant determinant of static and dynamic balance as well as quality of life in older community-dwelling women with osteoporosis. Gerontology 2002;48:360-368.

-10 Granacher U, Bridenbaugh SA, Muehlbauer T, Wehrle A, Kressig RW: Age-related effects on postural control under multi-task conditions. Gerontology 2011;57:247-255.

-11 Granacher U, Gruber M, Gollhofer A: Force production capacity and functional reflex activity in young and elderly men. Aging Clin Exp Res 2010;22:374-382.

- 12 Holviala JH, Sallinen JM, Kraemer WJ, Alen MJ, Hakkinen KK: Effects of strength training on muscle strength characteristics, functional capabilities, and balance in middleaged and older women. J Strength Cond Res 2006;20:336-344.
13 Izquierdo M, Aguado X, Gonzalez R, Lopez JL, Hakkinen K: Maximal and explosive force production capacity and balance performance in men of different ages. Eur J Appl Physiol Occup Physiol 1999;79:260-267.

14 Mayson DJ, Kiely DK, LaRose SI, Bean JF: Leg strength or velocity of movement: which is more influential on the balance of mobility limited elders? Am J Phys Med Rehabil 2008;87:969-976.

15 Melzer I, Benjuya N, Kaplanski J, Alexander $\mathrm{N}$ : Association between ankle muscle strength and limit of stability in older adults. Age Ageing 2009;38:119-123.

16 Menz HB, Morris ME, Lord SR: Foot and ankle characteristics associated with impaired balance and functional ability in older people. J Gerontol A Biol Sci Med Sci 2005;60: 1546-1552.

17 Shimada H, Obuchi S, Kamide N, Shiba Y, Okamoto M, Kakurai S: Relationship with dynamic balance function during standing and walking. Am J Phys Med Rehabil 2003; 82:511-516.

18 Woollacott M, Shumway-Cook A: Attention and the control of posture and gait: a review of an emerging area of research. Gait Posture 2002;16:1-14.

19 Lundin-Olsson L, Nyberg L, Gustafson Y: 'Stops walking when talking' as a predictor of falls in elderly people. Lancet 1997;349: 617.

20 Kapteyn TS, Bles W, Njiokiktjien CJ, Kodde L, Massen CH, Mol JM: Standardization in platform stabilometry being a part of posturography. Agressologie 1983;24:321-326.

21 Muller O, Gunther M, Krauss I, Horstmann T: Physical characterization of the therapeutic device Posturomed as a measuring device - presentation of a procedure to characterize balancing ability. Biomed Tech (Berl) 2004;49:56-60.

22 Montero-Odasso M, Schapira M, Varela C, Pitteri C, Soriano ER, Kaplan R, Camera LA, Mayorga LM: Gait velocity in senior people. An easy test for detecting mobility impairment in community elderly. J Nutr Health Aging 2004;8:340-343.
23 Podsiadlo D, Richardson S: The timed 'Up \& Go': a test of basic functional mobility for frail elderly persons. J Am Geriatr Soc 1991; 39:142-148.

-24 Duncan PW, Weiner DK, Chandler J, Studenski S: Functional reach: a new clinical measure of balance. J Gerontol 1990; 45:M192-197.

-25 Granacher U, Gruber M, Gollhofer A: Resistance training and neuromuscular performance in seniors. Int J Sports Med 2009;30: 652-657.

26 Newton RA: Validity of the multi-directional reach test: a practical measure for limits of stability in older adults. J Gerontol A Biol Sci Med Sci 2001;56:M248-252.

27 Beauchet O, Dubost V, Herrmann FR, Kressig RW: Stride-to-stride variability while backward counting among healthy young adults. J Neuroeng Rehabil 2005;2:26.

28 Laessoe U, Hoeck HC, Simonsen O, Voigt M: Residual attentional capacity amongst young and elderly during dual and triple task walking. Hum Mov Sci 2008;27:496-512.

29 Beauchet O, Dubost V, Aminian K, Gonthier R, Kressig RW: Dual-task-related gait changes in the elderly: does the type of cognitive task matter? J Mot Behav 2005;37:259-264.

30 Cohen J: A power primer. Psychol Bull 1992; 112:155-159.

31 Henwood TR, Riek S, Taaffe DR: Strength versus muscle power-specific resistance training in community-dwelling older adults. J Gerontol A Biol Sci Med Sci 2008;63: 83-91.

32 Kressig RW, Beauchet O: Guidelines for clinical applications of spatio-temporal gait analysis in older adults. Aging Clin Exp Res 2006;18:174-176. 